Pacific Journal of Mathematics

ON THE RIM-STRUCTURE OF CONTINUOUS IMAGES OF 


\title{
ON THE RIM-STRUCTURE OF CONTINUOUS IMAGES OF ORDERED COMPACTA
}

\author{
J. Nikiel, H. M. Tuncali, and E. D. Tymchatyn
}

Let $X$ be a Hausdorff continuous image of an ordered continuum. Mardešić proved that $X$ has a basis of open sets with metrizable boundaries. We use $T$-set approximations to obtain bases of open sets for $X$ whose boundaries satisfy a variety of conditions. In particular, we prove that

$$
\begin{aligned}
\operatorname{dim} X & =\operatorname{ind} X=\text { Ind } X \\
& =\max \{1, \sup \{\operatorname{dim} Y: Y \subset X \text { is metrizable and closed }\}\} .
\end{aligned}
$$

1. Introduction. In this paper we study the rim-properties of images of ordered continua and, more generally, of compact ordered spaces. Mardešić proved in [M1] that a Hausdorff space which is a continuous image of a compact ordered space is rim-metrizable. In [N3], the first author proved that every hereditarily locally connected continuum is a continuous image of an ordered continuum. Then he used the approximation by $\mathrm{T}$-sets of cyclic elements in images of ordered continua to prove that every hereditarily locally connected continuum is rim-countable. We use the techniques of [N3] to improve the result of Mardešić and to answer a question of Mardešić and Papić [MP] about dimension-theoretic properties of continuous images of ordered continua and ordered compacta. We improve a result of Simone [Si1] by proving that if $X$ is a continuous image of an ordered continuum and $X$ contains no nondegenerate metric continuum, then it is rim-finite. We also prove that if a rim-scattered space is a continuous image of an ordered compactum, then it is rim-countable.

All spaces in this paper are Hausdorff. A continuum is a compact connected (Hausdorff) space. An ordered compactum is a compact space which admits a linear ordering such that the order topology is the given topology. Ordered continua are locally connected; they are often called arcs.

A point $p$ of a connected set $X$ is a separating point of $X$ if $X-\{p\}$ is not connected. We let $E(X)$ denote the set of all separating points of $X$.

Let $X$ be a locally connected continuum. A connected subset $Q$ of $X$ is a cyclic element of $X$ if $Q$ is maximal with respect to containing 
no separating points of itself. Each cyclic element of $X$ is a locally connected continuum. The theory of cyclic elements is presented in [Wh1, Ch. 4] for the case of metric locally connected continua. We shall use some extensions of this theory to the non-metric setting as set out in [Wh2] and [C], see also [N4].

A collection $\mathbf{A}$ of subsets of a compact space $X$ is said to be a nullfamily in $X$ if, for every open covering $\mathbf{U}$ of $X$, the subcollection $\{B \in \mathbf{A}: B$ is not contained in any $V \in \mathbf{U}\}$ is finite.

Let $A$ be a subset of a locally connected continuum $X$. We let $K(X-A)$ denote the set of all components of $X-A$. We will say that $A$ is a T-set in $X$ if $A$ is closed and each component of $X-A$ has a two-point boundary.

Let $Y$ be a cyclic element of a locally connected continuum $X$. We say that a sequence $\left\{A_{1}, A_{2}, \ldots, A_{n}, \ldots\right\}$ of T-subsets of $Y$ T-approximates $Y$ if

(1) $A_{1}$ is metrizable,

(2) $A_{n} \subset A_{n+1}$,

(3) if $Z \in K\left(Y-A_{n}\right)$, then $E(\mathrm{Cl}(Z)) \subset A_{n+1}$,

(4) if $Z \in K\left(Y-A_{n}\right)$ and $C$ is a nondegenerate cyclic element of $\mathrm{Cl}(Z)$, then $C \cap A_{n+1}$ is a metrizable set which contains at least three points.

Note that the conditions of the above definition imply that $\mathrm{Cl}\left(\bigcup_{n=1}^{\infty} A_{n}\right)$ $=Y($ see $[\mathrm{N1}$, Lemma 3.4]).

In [N1], there are given several characterizations of continuous Hausdorff images of ordered continua. One of them is the following:

THEOREM 1 [N1, 1.1]. Let $X$ be a locally connected continuum. Then the following are equivalent:

(1) $X$ is a continuous image of an ordered continuum,

(2) if $Y$ is a nondegenerate cyclic element of $X$, then there is a sequence $\left\{A_{1}, A_{2}, \ldots\right\}$ of $T$-sets in $Y$ which $T$-approximates $Y$.

Further properties of continuous images of arcs and ordered compacta can be found in survey articles [M3], [TrW] and [N4]; see also [N1].

Let $\mathbf{P}$ be a property of sets. A space $X$ is said to be rim-P if it has a basis of open sets whose boundaries have property $\mathbf{P}$. A set is said to be scattered if each of its non-empty closed subsets has an 
isolated point. Recall that compact, metrizable, scattered spaces are countable. For definitions of dimensions dim, Ind and ind, the reader is referred to [E].

For a compact space $X$, we define

$$
\alpha(X)=\sup \{\operatorname{dim} Z: Z \text { is a closed metrizable subset of } X\} .
$$

We let $\alpha-1=\infty$ if $\alpha=\infty$.

We shall need the following lemmas.

LemMA 1 [Tr2]. Let $X$ be a locally connected continuum and $A$ a $T$-set in $X$. There exists an upper semi-continuous decomposition $G_{A}$ of $X$ into closed sets such that if $X_{A}$ denotes the quotient space and $f: X \rightarrow X_{A}$ is the quotient map, then:

(1) $\left.f\right|_{A}$ is one-to-one and $f(A)$ is a T-set in $X_{A}$,

(2) each $Z \in K\left(X_{A}-f(A)\right)$ is homeomorphic to ]0, 1[,

(3) for each $Z \in K\left(X_{A}-f(A)\right)$ there exists a unique $P_{Z} \in K(X-A)$ such that $f\left(P_{Z}\right) \subset \mathrm{Cl}(Z)$, and each component of $X-A$ is a $P_{Z}$ for some $Z \in K\left(X_{A}-f(A)\right)$.

In the above lemma, $f(A)$ is a T-set in $X_{A}$, and we call $f$ a T-map with respect to $A$. The space $X_{A}$ is uniquely determined by $X$ and $A$. If the set $A$ is metrizable it follows, by local connectedness of $X$, that $K(X-A)$ is countable, [N1, 4.1].

LEMMA 2. Let $X$ be a locally connected continuum and, for every cyclic element $Y$ of $X$, let $\mathbf{B}_{Y}$ be a basis for $Y$. Then $X$ has a basis B such that, for each $U \in \mathbf{B}$, there exist a family $\mathbf{A}$ of cyclic elements of $X$, non-negative integers $m$ and $n$, nondegenerate cyclic elements $Y_{1}, \ldots, Y_{m}$ of $X$, sets $U_{1} \in \mathbf{B}_{Y_{1}}, \ldots, U_{m} \in \mathbf{B}_{Y_{m}}$, and separating points $x_{1}, \ldots, x_{n}$ of $X$ such that

$$
\begin{gathered}
U=(\bigcup \mathbf{A}) \cup U_{1} \cup \cdots \cup U_{m} \text { and } \\
\operatorname{Bd}(U)=\operatorname{Bd}_{Y_{1}}\left(U_{1}\right) \cup \cdots \cup \operatorname{Bd}_{Y_{m}}\left(U_{m}\right) \cup\left\{x_{1}, \ldots, x_{n}\right\} .
\end{gathered}
$$

Proof. The lemma follows from the generalization, by Cornette [C, p. 225-6], of Whyburn's cyclic chain approximation theorem [Wh1, IV.7.1, p. 73] to the case of locally connected Hausdorff continua.

LEMMA 3. Let $\gamma$ be an infinite cardinal number and let $\mathbf{P}$ be a hereditary property of compact sets that is preserved under unions of fewer than $\gamma$ compact sets. Let $X$ be a locally connected continuum, 
$\left\{A_{i}\right\}_{i=1}^{\infty}$ an increasing sequence of closed subsets of $X$, and $\left\{\mathbf{V}_{i}\right\}_{i=1}^{\infty} a$ sequence of collections of sets such that:

(1) $\mathbf{V}_{i}$ is a basis of open sets for $A_{i}$,

(2) $\operatorname{Bd}(K)$ has property $\mathbf{P}$ for each $K \in K\left(X-A_{i}\right)$,

(3) $V \in \mathbf{V}_{i}$ implies $\mathrm{Bd}_{A_{i}}(V)$ has property $\mathbf{P}$,

(4) $V \in \mathbf{V}_{i}$ implies $\left\{K \in K\left(X-A_{i}\right): \operatorname{Bd}(K) \cap V \neq \varnothing\right.$ and $\operatorname{Bd}(K)$ $\not \subset \mathrm{Cl}(V)\}$ has cardinality less than $\gamma$,

(5) for each open cover $\mathbf{W}$ of $X$ there is an integer $i$ such that $K\left(X-A_{i}\right)$ refines $\mathbf{W}$.

Then $X$ admits a basis of open sets whose boundaries have property P.

Proof. Let $x \in X$ and let $U$ be an open neighbourhood of $x$. Let $W$ be an open neighbourhood of $x$ such that $\mathrm{Cl}(W) \subset U$.

Suppose that $x \notin \bigcup_{n=1}^{\infty} A_{n}$. For every $n$ let $K_{n} \in K\left(X-A_{n}\right)$ be such that $x \in K_{n}$. Then $K_{n+1} \subset K_{n}$. By (5), there is an integer $i$ such that $K_{i}$ is contained either in $U$ or in $X-\mathrm{Cl}(W)$. Since $x \in \mathrm{Cl}(W) \cap K_{i}$, it follows that $K_{i} \subset U$. Since $X$ is locally connected, $K_{i}$ is an open set. $\mathrm{By}(2), \operatorname{Bd}\left(K_{i}\right)$ has property $\mathbf{P}$.

Now suppose that $x \in A_{n}$ for some integer $n$. By (5), we may take $n$ to be such that no component of $X-A_{n}$ meets both $\mathrm{Cl}(W)$ and $X-U$. Let $V \in \mathrm{V}_{n}$ be such that $x \in V \subset \mathrm{Cl}(V) \subset W$. Let $V^{\prime}=V \cup \bigcup\left\{K \in K\left(X-A_{n}\right): \operatorname{Bd}(K) \cap V \neq \varnothing\right\}$. Then $V^{\prime} \subset U$. Since $X$ is locally connected, $V^{\prime}$ is open and

$\operatorname{Bd}\left(V^{\prime}\right) \subset \operatorname{Bd}_{A_{n}}(V)$

$\cup \bigcup\left\{\operatorname{Bd}(K): K \in K\left(X-A_{n}\right), \operatorname{Bd}(K) \cap V \neq \varnothing\right.$ and $\left.\operatorname{Bd}(K) \not \subset V\right\}$. By (3), (2) and (4), it follows that $\operatorname{Bd}\left(V^{\prime}\right)$ has property $\mathbf{P}$.

2. Main results. The proof of the following lemma uses some ideas from the proof of [N3, Theorem 4.1].

LEMMA 4. Let $Y$ be a continuum with no separating point which is a continuous image of an ordered continuum. Let $\alpha=\max \{1, \alpha(Y)\}$. Then $Y$ has a basis $\mathbf{V}$ of open sets whose boundaries are metrizable sets of $\operatorname{dim} \leq \alpha-1$. Moreover, if $Y$ admits a basis of open sets with scattered boundaries, then the boundaries of members of $\mathbf{V}$ are countable.

Proof. Let $\left\{A_{1}, A_{2}, \ldots\right\}$ be a sequence of T-sets in $Y$ which Tapproximates $Y$. For each $n$, let $f_{n}: Y \rightarrow Y_{A_{n}}=Y_{n}$ be a T-map with 
respect to $A_{n}$ (see Lemma 1). We let $B_{n}^{m}=f_{n}\left(A_{m}\right) \subset Y_{n}$ provided $m \leq n$. Notice that $Y_{n}$ has no separating point, each $B_{n}^{m}$ is a Tset in $Y_{n}$ provided $m \leq n,\left.f_{n}\right|_{A_{m}}: A_{m} \rightarrow B_{n}^{m}$ is a homeomorphism, and every component of $Y_{n}-B_{n}^{n}$ is homeomorphic to ]0, 1[. Since $Y_{n}$ has no separating point, it follows that if $P$ is a component of $Y_{n}-B_{n}^{m}, \operatorname{Bd}(P)=\{a, b\}$, then $\mathrm{Cl}(P)$ is a cyclic chain from $a$ to $b$ (in the case when $m=n-1$, all cyclic elements of $\mathrm{Cl}(P)$ are metrizable-see below).

First, we use an induction to show that, for $n=1,2, \ldots, Y_{n}$ has a basis $\mathbf{B}_{n}$ such that $\operatorname{Bd}_{Y_{n}}(V)$ is metrizable and $\operatorname{dim}\left(\operatorname{Bd}_{Y_{n}}(V)\right) \leq \alpha-1$ for each $V \in \mathbf{B}_{n}$.

Note that $Y_{1}=B_{1}^{1} \cup\left(Y_{1}-B_{1}^{1}\right)$ is a metrizable space which is a union of the compact metrizable set $B_{1}^{1}$ (which is homeomorphic to $A_{1}$ ) and a countable family of copies of $] 0,1[$. By [E, 1.5.3, p. 42], $\operatorname{dim} Y_{1} \leq \max \left\{1, \operatorname{dim} B_{1}^{1}\right\} \leq \alpha$. Hence, $Y_{1}$ has a basis $\mathbf{B}_{1}$ as required.

Suppose that the required basis $\mathbf{B}_{n}$ for $Y_{n}$ has been already defined. Let $y \in Y_{n+1}$ and let $V$ be an open neighbourhood of $y$ in $Y_{n+1}$. If $y \notin B_{n+1}^{n}$, then $y \in Q$ for some $Q \in K\left(Y_{n+1}-B_{n+1}^{n}\right)$. Let $\operatorname{Bd}(Q)=\{a, b\}$. Then $\operatorname{Cl}(Q)$ is a cyclic chain from $a$ to $b$ and $E(\mathrm{Cl}(Q)) \subset B_{n+1}^{n+1}$. If $Z$ is a nondegenerate cyclic element of $\mathrm{Cl}(Q)$, then $B_{Z}=B_{n+1}^{n+1} \cap Z$ is a metrizable T-set in $Z, Z \cap(E(C l(Q)) \cup$ $\{a, b\})$ consists of exactly two points, and each component of $Z-B_{Z}$ is homeomorphic to ]0, 1 [. Hence, $K\left(Z-B_{Z}\right)$ is countable and $Z$ is metrizable. Now, it is easy to find an open neighbourhood $W$ of $y$ in $Y_{n+1}$ such that $W \subset V \cap Q, \operatorname{Bd}_{Y_{n}}(W)$ is contained in two cyclic elements $Z_{1}$ and $Z_{2}$ of $\mathrm{Cl}(Q)$ and for $i=1,2$

$$
\begin{aligned}
\operatorname{dim}\left(\operatorname{Bd}_{Y_{n}}(W) \cap Z_{i}\right) & \leq \operatorname{dim} Z_{i}-1 \leq \max \left\{1, \operatorname{dim} B_{Z_{i}}\right\}-1 \\
& \leq \max \left\{1, \operatorname{dim} A_{n+1}\right\}-1 \leq \alpha-1
\end{aligned}
$$

provided $Z_{i}$ is nondegenerate (the case when $Z_{i}$ is degenerate is trivial). Thus we have $\operatorname{dim}\left(\operatorname{Bd}_{Y_{n}}(W)\right) \leq \alpha-1$.

Now, suppose that $y \in B_{n+1}^{n}$. Let $x$ denote the unique point of $A_{n}$ such that $f_{n+1}(x)=y$. For every $P \in K\left(Y_{n+1}-B_{n+1}^{n}\right)$ let $Q_{P} \in$ $K\left(Y-A_{n}\right)$ be a component such that $f_{n+1}\left(Q_{P}\right) \subset \mathrm{Cl}(P)$ and let $R_{P} \in$ $K\left(Y_{n}-B_{n}^{n}\right)$ be such that $f_{n}\left(Q_{P}\right) \subset \mathrm{Cl}\left(R_{P}\right)$. Set $\operatorname{Bd}_{Y_{n+1}}(P)=\left\{a_{P}, b_{P}\right\}$ and $\operatorname{Bd}_{Y_{n}}\left(R_{P}\right)=\left\{a_{P}^{\prime}, b_{P}^{\prime}\right\}$, where $f_{n+1}^{-1}\left(a_{n}\right) \cap A_{n}=f_{n}^{-1}\left(a_{n}^{\prime}\right) \cap A_{n}$, and let $\leq$ denote the natural ordering on $\mathrm{Cl}\left(R_{P}\right)$ from $a_{P}^{\prime}$ to $b_{P}^{\prime}$. Choose $r_{P} \in R_{P}$ and let $I_{P}=\left\{r \in R_{P}: r<r_{P}\right\}$ and $J_{P}=\left\{r \in R_{P}: r_{P}<r\right\}$. 
Let

$$
\begin{aligned}
V^{\prime}= & f_{n}\left(f_{n+1}^{-1}(V) \cap A_{n}\right) \\
& \cup \bigcup\left\{R_{P}: P \in K\left(Y_{n+1}-B_{n+1}^{n}\right) \text { and } \mathrm{Cl}(P) \subset V\right\} \\
& \cup \bigcup\left\{I_{P}: P \in K\left(Y_{n+1}-B_{n+1}^{n}\right) \text { and } a_{P} \in V\right\} \\
& \cup \bigcup\left\{J_{P}: P \in K\left(Y_{n+1}-B_{n+1}^{n}\right) \text { and } b_{P} \in V\right\} .
\end{aligned}
$$

Since $\left\{\mathrm{Cl}\left(R_{P}\right): P \in K\left(Y_{n+1}-B_{n+1}^{n}\right)\right\}$ is a null-family, $V^{\prime}$ is an open subset of $Y_{n}$. Moreover, $f_{n}(x) \in V^{\prime}$. By the inductive hypothesis, there is a connected open set $W^{\prime}$ in $Y_{n}$ such that $f_{n}(x) \in W^{\prime} \subset V^{\prime}$, $\operatorname{Bd}_{Y_{n}}\left(W^{\prime}\right)$ is metrizable and $\operatorname{dim}\left(\operatorname{Bd}_{Y_{n}}\left(W^{\prime}\right)\right) \leq \alpha-1$. Let

$$
\begin{aligned}
& \mathbf{H}_{1}=\left\{P \in K\left(Y_{n+1}-B_{n+1}^{n}\right): a_{P}^{\prime} \in W^{\prime} \text { and } R_{P} \not \subset W^{\prime}\right\}, \\
& \mathbf{H}_{2}=\left\{P \in K\left(Y_{n+1}-B_{n+1}^{n}\right): b_{P}^{\prime} \in W^{\prime} \text { and } R_{P} \not \subset W^{\prime}\right\}
\end{aligned}
$$

and

$$
\mathbf{H}_{3}=\left\{P \in K\left(Y_{n+1}-B_{n+1}^{n}\right): R_{P} \subset W^{\prime}\right\} .
$$

Note that if $P \in \mathbf{H}_{1} \cup \mathbf{H}_{2}$, then $R_{P} \cap \mathrm{Bd}_{Y_{n}}\left(W^{\prime}\right)$ is a non-empty open subset of $\mathrm{Bd}_{Y_{n}}\left(W^{\prime}\right)$. Since $\mathrm{Bd}_{Y_{n}}\left(W^{\prime}\right)$ is compact and metrizable, $\mathbf{H}_{1} \cup$ $\mathbf{H}_{2}$ is countable. For every $P \in \mathbf{H}_{1}$ (resp. $P \in \mathbf{H}_{2}$ ), let $W_{P}^{1}$ (resp. $W_{P}^{2}$ ) be an open subset of $\mathrm{Cl}(P)$ such that $a_{P} \in W_{P}^{1} \subset V$ (resp. $b_{P} \in$ $\left.W_{P}^{2} \subset V\right), \operatorname{Bd}_{\mathrm{Cl}(P)}\left(W_{P}^{1}\right)$ is metrizable and $\operatorname{dim}\left(\mathrm{Bd}_{\mathrm{Cl}(P)}\left(W_{P}^{1}\right)\right) \leq \alpha-1$ (resp. $\operatorname{Bd}_{\mathrm{Cl}(P)}\left(W_{P}^{2}\right)$ is metrizable and $\left.\operatorname{dim}\left(\mathrm{Bd}_{\mathrm{Cl}(P)}\left(W_{P}^{2}\right)\right) \leq \alpha-1\right)$. Note that $\mathrm{Bd}_{\mathrm{Cl}(P)}\left(W_{P}^{i}\right)$ may be assumed to be contained in one cyclic element $Z$ of $\mathrm{Cl}(P)$. By the fact that $K\left(Z-B_{Z}\right)$ is countable, it follows that $Z$ is metrizable and $\operatorname{dim} Z \leq \alpha$. Let

$$
W=f_{n+1}\left(f_{n}^{-1}\left(W^{\prime}\right) \cap A_{n}\right) \cup \bigcup_{P \in \mathbf{H}_{1}} W_{P}^{1} \cup \bigcup_{P \in \mathbf{H}_{2}} W_{P}^{2} \cup \bigcup \mathbf{H}_{3} .
$$

Since $K\left(Y_{n+1}-B_{n+1}^{n}\right)$ is a null-family, $W$ is open in $Z$. A straightforward argument shows that $y \in W \subset V$ (because if $P \in K\left(Y_{n+1}-B_{n+1}^{n}\right)$ is not contained in $V$, then $r_{P} \notin V^{\prime}$ and so $\left.R_{P} \not \subset W^{\prime}\right)$ and

$$
\begin{aligned}
\operatorname{Bd}_{Y_{n+1}}(W)= & f_{n+1}\left(f_{n}^{-1}\left(\operatorname{Bd}_{Y_{n}}\left(W^{\prime}\right) \cap A_{n}\right)\right) \\
& \cup \bigcup_{P \in \mathbf{H}_{1}} \operatorname{Bd}_{\mathrm{Cl}(P)}\left(W_{P}^{1}\right) \cup \bigcup_{P \in \mathbf{H}_{2}} \operatorname{Bd}_{\mathrm{Cl}(P)}\left(W_{P}^{2}\right) .
\end{aligned}
$$

Thus $\operatorname{Bd}_{Y_{n+1}}(W)$ is a union of countably many compact metrizable sets of $\operatorname{dim} \leq \alpha-1$. It is well-known that each compact space which 
can be covered by countably many closed and metrizable subsets is metrizable. Hence, $\operatorname{Bd}_{Y_{n+1}}(W)$ is metrizable. By [E, 1.5.3, p. 42], $\operatorname{dim}\left(\operatorname{Bd}_{Y_{n+1}}(W)\right) \leq \alpha-1$. The inductive argument is complete.

Let $\mathbf{P}^{n+1}$ be the following property of compact spaces: a space is metrizable of dimension $\leq \alpha-1$. Let $\gamma=\aleph_{1}$ be the first uncountable cardinal number. Note that $Y$ satisfies all the assumptions of Lemma 3. Indeed, the condition (2) of Lemma 3 follows immediately from the definition of a T-set. Let $\mathbf{V}_{n}=\left\{A_{n} \cap f_{n}^{-1}(U): U \in \mathbf{B}_{n}\right\}$ for $n=1,2, \ldots$. Then $\mathbf{V}_{n}$ is a basis for $A_{n}$ which satisfies the conditions (1) and (3). The condition (4) follows from [N1, 4.1], and the condition (5) is a consequence of [N1, 3.4]. By Lemma 3, $Y$ has a basis $\mathbf{V}$ of open sets with metrizable boundaries of dimension $\leq \alpha-1$.

Suppose that $Y$ is rim-scattered. Then $Y_{1}$ is metrizable and rimscattered. Hence, $Y_{1}$ has a basis of open sets with countable boundaries. It is now easy to modify the above argument to show that each $Y_{n}$ has a basis of open sets with countable boundaries. By Lemma 3, $Y$ has a basis of open sets with countable boundaries.

Simone, [Si1] and [Si2], proved that if $X$ is a continuum with degree of cellularity $\aleph_{0}$, which is a continuous image of an ordered continuum and which contains no nondegenerate metric subcontinuum, then $X$ has a basis of open sets with finite boundaries. Simone's theorem can be improved as follows:

THEOREM 2. Let $X$ be a continuum which is a continuous image of an arc and which contains no nondegenerate metric subcontinuum. Then $X$ has a basis of open sets with finite boundaries.

Proof. Let $Y$ be a nondegenerate cyclic element of $X$. Since having a basis of open sets with finite boundaries is a cyclically extensible property (see Lemma 2), it suffices to prove that $Y$ is rim-finite.

Let $\left\{A_{1}, A_{2}, \ldots\right\}$ be a sequence of T-sets in $Y$ which T-approximates $Y$ and, for $n=1,2, \ldots$, let $f_{n}: Y \rightarrow Y_{n}$ be a T-map with respect to $A_{n}$ (see Lemma 1 ). Since $A_{1}$ is metrizable, and, hence, zero-dimensional, $Y_{1}$ has a basis of open sets with finite boundaries (see [N1, 4.3]). If $U$ is an open set in $Y_{1}$ which has a finite boundary, then all but at most finitely many components of $Y_{1}-A_{1}$ whose closures meet $U \cap A_{1}$ are contained in $\mathrm{Cl}(U)$. An inductive argument similar to the one given in the proof of Lemma 4 shows that each $Y_{n}$ is rim-finite. Taking $\mathbf{P}$ to be the property of being a finite set and 
$\gamma=\aleph_{0}$ in Lemma 3, it follows that $Y$ has a basis of open sets with finite boundaries.

THEOREM 3. If $X$ is a nondegenerate continuous image of an ordered continuum, then

$$
\max \{1, \alpha(X)\}=\operatorname{dim} X=\operatorname{Ind} X=\operatorname{ind} X .
$$

Proof. Let $\alpha=\max \{1, \alpha(X)\}$. Since $X$ is a nondegenerate continuum, ind $X \geq 1$. By general facts (see [E, 3.1.4 on p. 209, 2.2.1 on p. 170, and 1.1.2 on p. 4]), it follows that $\operatorname{dim} X \geq \operatorname{dim} Z$, Ind $X \geq$ Ind $Z$ and ind $X \geq$ ind $Z$ for each closed subspace $Z$ of $X$. Hence $\operatorname{dim} X$, Ind $X$, ind $X \geq \alpha$. For each normal space $X$, we have ind $X \leq \operatorname{Ind} X[\mathbf{E}, 1.6 .3$, p. 52] and $\operatorname{dim} X \leq \operatorname{Ind} X[\mathbf{E}, 3.1 .28$, p. 220]. Thus it suffices to show that Ind $X \leq \alpha$.

Let $x \in X$ and $V$ be an open neighbourhood of $x$. By Lemmas 4 and 2 , there exists an open set $W$ such that $x \in W \subset V, \operatorname{Bd}(W)$ is contained in the union of a finite collection $\left\{Z_{1}, \ldots, Z_{n}\right\}$ of cyclic elements of $X, \operatorname{Bd}(W) \cap Z_{i}$ is metrizable and $\operatorname{dim}\left(\operatorname{Bd}(W) \cap Z_{i}\right) \leq \alpha-1$ for $i=1, \ldots, n$. Hence, $\operatorname{Bd}(W)$ is metrizable and $\operatorname{Ind} \operatorname{Bd}(W)=$ $\operatorname{dim} \operatorname{Bd}(W) \leq \alpha-1$. By the sum theorem for separable metric spaces, [E, 1.5.3, p. 42], we have Ind $X \leq \alpha$.

REMARK. In Theorem 3, if $\alpha(X)=0$, then $X$ is rim-finite by Theorem 2.

THEOREM 4. Let $X$ be a continuum which is a continuous image of an arc. If $X$ has a basis of open sets with scattered boundaries, then it has a basis of open sets with countable boundaries.

Proof. By Lemma 4, each cyclic element of $X$ is rim-countable. The theorem follows by Lemma 2.

The following theorem answers a question of Mardešić and Papić ([MP], see also [N4, Problem 4]):

THEOREM 5. Let $Z$ be a continuous image of a compact ordered space. Then

(1) $\operatorname{dim} Z=\operatorname{Ind} Z=$ ind $Z$. If, moreover, $\operatorname{dim} Z>0$ then $\operatorname{dim} Z=\max \{1, \alpha(Z)\}$.

(2) If $Z$ is rim-scattered, then it is rim-countable.

Proof. For every compact space $T$, Ind $T=0$ iff $\operatorname{dim} T=0$ iff ind $T=0,[\mathbf{E}, 3.1 .30, \mathrm{p}$. 221]. Thus we may assume that $Z$ is not zero-dimensional. Let $\alpha=\max \{1, \alpha(Z)\}$. 
By [N2, Theorem 2], see also [M1, Lemma 8], there exists a space $X$ such that $X$ is a continuous image of an $\operatorname{arc}, Z \subset X, Z$ is a T-set in $X$, and each component of $X-Z$ is homeomorphic to ]0, 1 [. If $Y$ is a closed metrizable subset of $X$, then $Y$ is a union of $Z \cap Y$ and at most countably many closed sets which are homeomorphic to subsets of $] 0,1[$. Hence, $\operatorname{dim} Y \leq \max \{1, \operatorname{dim}(Y \cap Z)\}$. By Theorem 3, $\alpha=\operatorname{dim} X=\operatorname{Ind} X=\operatorname{ind} X$. Since $Z$ is not zerodimensional, $\alpha \leq \operatorname{dim} Z$, Ind $Z$, ind $Z$. However, $\operatorname{dim} Z \leq \operatorname{dim} X$, Ind $Z \leq$ Ind $X$ and ind $Z \leq$ ind $X$. This completes the proof of (1). A similar argument together with Theorem 4 show that (2) holds.

REMARKS. 1. In the case when $\alpha(Z)=0$, the result (1) of Theorem 5 was obtained by Mardešić [M2, Corollary, p. 425].

2. The proofs of Lemma 4 and Theorems 3 and 5 show that if a space $X$ is a continuous image of an ordered compactum, then it has a basis $B$ such that $\operatorname{Bd}(U)$ is metrizable and $\operatorname{dim} \operatorname{Bd}(U) \leq \operatorname{dim} X-1$ for each $U \in \mathbf{B}$. This improves results of [M1].

3. Problems. Filippov gave in [F] an example of a locally connected continuum which admits a basis of open sets with metrizable zerodimensional and perfect boundaries and which is not a continuous image of any ordered compactum.

In general, rim-scattered continua are not continuous images of ordered compacta. For example: the space $X=L \times S /\{0\} \times S$, where $L$ denotes the long interval and $S=\left\{\frac{1}{n}: n=1,2, \ldots\right\} \cup\{0\}$, is a rimcountable continuum which is a continuous image of no ordered compactum. In fact, $X$ contains a non-metric product of infinite compact spaces-see [Tr1]. However, the space $X$ is not locally connected. In [Tu], it was proved that rim-scattered locally connected continua do not contain a non-metric product of nondegenerate continua. Hence we may ask the following question:

Question 1. Is every locally connected rim-scattered continuum a continuous image of an ordered continuum?

Filippov's example shows that rim-scattered locally connected continua are the largest possible class of spaces defined with the use of rim-properties that could be contained in the class of continuous images of ordered continua. Recall the following weaker question which is still open (see [N3] and [N4]).

Question 2. Is every locally connected, rim-countable continuum a continuous image of an ordered continuum? 
Let us also pose the following problem:

Question 3. Is every locally connected and rim-scattered continuum a rim-countable space?

Recall that, by Theorem 4, Question 3 has a positive answer provided the space under consideration is a continuous image of an arc.

Added in proof. Recently the authors answered questions 1 and 2 in the negative in the paper: J. Nikiel, H. M. Tuncali, and E. D. Tymchatyn, A locally connected rim-countable continuum which is the continuous image of no arc, Topology Appl. (to appear). L. B. Treybig proved a result which implies Theorem 2 in Proc. Amer. Math. Soc. 74 (1979), 326-328.

\section{REFERENCES}

[C] J. L. Cornette, "Image of a Hausdorff arc" is cyclically extensible and reducible, Trans. Amer. Math. Soc., 199 (1974), 253-267.

[E] R. Engelking, Dimension theory, North-Holland and PWN-Polish Scientific Publishers, 1978.

[F] V. V. Filippov, On perfectly normal compacta, in Russian, Dokl. Akad. Nauk SSSR, 189 (1969), 736-739.

[M1] S. Mardešić, Images of ordered compacta are locally peripherally metric, $\mathrm{Pa}-$ cific J. Math., 23 (1967), 557-568.

[M2] _- Continuous images of ordered compacta and a new dimension which neglects metric subcontinua, Trans. Amer. Math. Soc., 121 (1966), 424-433.

[M3] - On the Hahn-Mazurkiewicz theorem in non-metric spaces, in General Topology and its Relations to Modern Analysis and Algebra II, Prague (1966), 248-255.

[MP] S. Mardešić and P. Papić, Some problems concerning mappings of ordered compacta, in Serbo-Croatian, English summary, Matematička Biblioteka, 25 (1963), 11-22.

[N1] J. Nikiel, Images of arcs-a nonseparable version of the Hahn-Mazurkiewicz theorem, Fund. Math., 129 (1988), 91-120.

[N2] _- Some problems on continuous images of compact ordered spaces, Questions Answers Gen. Topology, 4 (1986/87), 117-128.

[N3] _ The Hahn-Mazurkiewicz theorem for hereditarily locally connected continua, Topology Appl., 32 (1989), 307-323.

[N4] _- On continuous images of arcs and compact orderable spaces, Topology Proceedings, 14 (1989), 163-193.

[Si1] J. N. Simone, Metric components of continuous images of ordered compacta, Pacific J. Math., 69 (1977), 269-274.

[Si2] _ Continuous images of ordered compacta and hereditarily locally connected continua, Colloq. Math., 40 (1978), 77-84.

[Tr1] L. B. Treybig, Concerning continuous images of compact ordered spaces, Proc. Amer. Math. Soc., 15 (1964), 866-871. 
[Tr2] L. B. Treybig, Arcwise connectivity in continuous images of ordered compacta, Glasnik Mat., 21 (1986), 201-211.

[TrW] L. B. Treybig and L. E. Ward, Jr., The Hahn-Mazurkiewicz problem, in Topology and order structure, part 1, Math. Centre Tracts, vol. 142. Amsterdam, 1981, pp. 95-105.

[Tu] H. M. Tuncali, Analogues of Treybig's product theorem, Proc. Amer. Math. Soc., 108 (1990), 855-858.

[Wh1] G. T. Whyburn, Analytic Topology, Amer. Math. Soc., Providence, R. I., 1942.

[Wh2] _ Cut points in general topological spaces, Proc. Nat. Acad. Sci. USA, 61 (1968), 380-387.

Received January 26, 1990. This research was supported in part by NSERC grant number A5616 and by a grant from the University of Saskatchewan President's Fund.

UNIVERSITY OF SASKATCHEWAN

SASKaTOON, SASKaTCHEWAN

CANADA S7N 0W0 



\section{PACIFIC JOURNAL OF MATHEMATICS EDITORS}

\author{
V. S. VARADARAJAN \\ (Managing Editor) \\ University of California \\ Los Angeles, CA 90024-1555-05 \\ Herbert Clemens \\ University of Utah \\ Salt Lake City, UT 84112 \\ THOMAS ENRIGHT \\ University of California, San Diego \\ La Jolla, CA 92093
}

R. FINN

Stanford University

Stanford, CA 94305

HeRmann FlaschKa

University of Arizona

Tucson, AZ 85721

VAUGHAN F. R. JoNES

University of California

Berkeley, CA 94720

STEVEN KERCKHOFF

Stanford University

Stanford, CA 94305
C. C. MOORE

University of California

Berkeley, CA 94720

MaRTIN SCHARLEMANN

University of California

Santa Barbara, CA 93106

HAROLD STARK

University of California, San Diego

La Jolla, CA 92093

\section{ASSOCIATE EDITORS}
R. ARENS
E. F. BECKENBACH
B. H. NeumanN
F. WolF
K. YosHIDA

\section{SUPPORTING INSTITUTIONS}

UNIVERSITY OF ARIZONA

UNIVERSITY OF BRITISH COLUMBIA

CALIFORNIA INSTITUTE OF TECHNOLOGY

UNIVERSITY OF CALIFORNIA

MONTANA STATE UNIVERSITY

UNIVERSITY OF NEVADA, RENO

NEW MEXICO STATE UNIVERSITY

OREGON STATE UNIVERSITY
UNIVERSITY OF OREGON

UNIVERSITY OF SOUTHERN CALIFORNIA

STANFORD UNIVERSITY

UNIVERSITY OF HAWAII

UNIVERSITY OF TOKYO

UNIVERSITY OF UTAH

WASHINGTON STATE UNIVERSITY

UNIVERSITY OF WASHINGTON 


\section{Pacific Journal of Mathematics}

Vol. 149, No. $1 \quad$ May, 1991

Takao Akahori and Harunori Ameku, On the Romanov kernel and Kuranishi's $L^{2}$-estimate for $\bar{\partial}_{\mathrm{b}}$ over a ball in the strongly pseudo convex

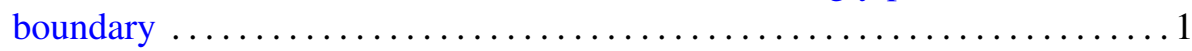

Robert Emile Beaudoin, The proper forcing axiom and stationary set

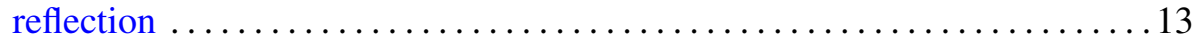

Hans Engler, A matrix Volterra integrodifferential equation occurring in

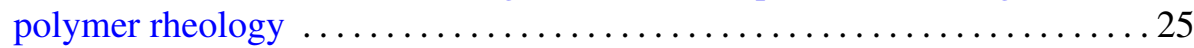

Zhong Ge, On a constrained variational problem and the spaces of

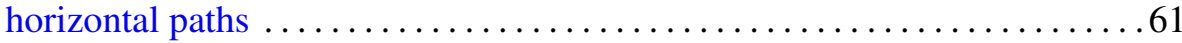

Yutaka Hemmi, Higher homotopy commutativity of $H$-spaces and the

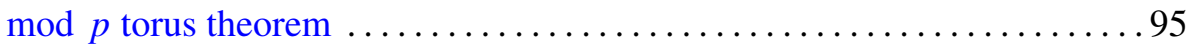

G. D. Johnson, An intrinsic characterization of a class of minimal surfaces

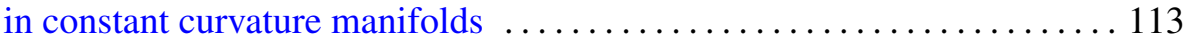

Min Ho Lee, Conjugates of equivariant holomorphic maps of symmetric domains

Jacek Nikiel, H. Murat Tuncali and Edward D. Tymchatyn, On the rim-structure of continuous images of ordered compacta

Tara Lynn Smith, Generalized Clifford-Littlewood-Eckmann groups . . . . . 157

Tara Lynn Smith, Generalized Clifford-Littlewood-Eckmann groups II:

Linear representations and applications 185 